\title{
Tensile deformation behaviour of ferritic-pearlitic steel studied by digital image correlation method
}

\author{
M. Štamborská ${ }^{*}$, J. Lapin ${ }^{1}$, O. Bajana ${ }^{1}$, M. Losertová ${ }^{2}$ \\ ${ }^{1}$ Institute of Materials \& Machine Mechanics, Slovak Academy of Sciences, \\ Račianska 75, 83102 Bratislava, Slovak Republic \\ ${ }^{2} V \breve{S} B$ - Technical University of Ostrava, Faculty of Metallurgy and Materials Engineering, Department of Non-Ferrous \\ Metals, Refining and Recycling, Ostrava - Poruba, Czech Republic
}

Received 20 June 2015, received in revised form 3 September 2015, accepted 7 September 2015

\begin{abstract}
Tensile deformation behaviour of ferritic-pearlitic steel was studied by the digital image correlation (DIC) method. Room temperature tensile tests were carried out at an initial strain rate of $1 \times 10^{-3} \mathrm{~s}^{-1}$ up to a defined elongation or fracture. The elongation and contraction were measured on the surface of cylindrical tensile specimens with random speckle pattern using stereo CCD camera system. The DIC elongation data were related to the data measured by an extensometer touching the specimens. The data from the DIC method were used for calculations of true stress-true strain tensile curves, strain hardening exponents and work hardening rates during uniform deformation up to the onset of plastic instability as well as non-uniform deformation during necking. Numerical simulations of strain fields within the gauge region of the specimens using finite element method were validated by the experimental data from the DIC method.
\end{abstract}

K e y w o r d s: work hardening rate, ferritic-pearlitic steel, finite element method, digital image correlation

\section{Introduction}

Ferritic-pearlitic steels are widely used in industry for many engineering applications. This group of materials includes a wide range of plain carbon steels where alloying additions are primarily made for steel-making purposes. For example, manganese is added to combine with sulphur, but it is also a strengthener while manganese and silicon are deoxidizers [1]. Ferrite-pearlite microstructures are also found in the intermediary state of the hot-rolled strips required for the production of the newest high-strength steels such as dual phase (DP) and transformation induced plasticity (TRIP) steels. A major part of the hot strip production of steel makers is directly delivered to customers and post-processors with ferrite-

-pearlite microstructure. These semi products are usually subjected to input material inspection including tensile testing at room temperature. Extensive studies have been conducted on the effect of microstructure on the tensile deformation behaviour of ferritic-pearlitic steels. However, classical tensile tests can provide only limited information about deformation behaviour of these materials.

Tensile specimens of metallic materials experience elastic deformation, plastic yielding, necking instability, and neck growth eventually leading to fracture due to a decrease in the load bearing capability. In order to obtain the correct mechanical properties and understand the tensile behaviour related to the intrinsic properties of materials, not only experiments using precise machines and sensors but also interpretation of the flow curves resulting from the combination of the intrinsic mechanical properties, specimen geometries, and testing conditions is important. Contactless sensing digital image correlation (DIC) optical method for tracking and image registration technique allows accurate 3D measurements of gauge section of specimens during tensile testing and can provide new

\footnotetext{
*Corresponding author: tel.: +421 59309 217; e-mail address: stamborska.michaela@gmail.com
} 
information about tensile deformation behaviour of materials [2-4]. The additional data obtained during testing can be used to understand more deeply whole tensile deformation process including calculations of local elongation, local contraction and redistribution of strain fields on the surface of the specimen. Hence, application of the DIC method for tensile testing of classical ferritic-pearlitic steels is of great interest to extend the existing knowledge about the deformation behaviour of this widely used group of materials.

The aim of this article is to study deformation behaviour of ferritic-pearlitic steel and to apply DIC method for tracking, image registration and 3D measurements of changes in gauge section of specimens during tensile testing at room temperature. The achieved experimental results are supported by numerical calculations of the strain fields on the surface of tensile specimens using experimental data from DIC measurements as well as redistribution of strains within the gauge section using finite element analysis (FEA).

\section{Experimental procedure}

The studied material was received in the form of cylindrical bars with a diameter of $10 \mathrm{~mm}$. The chemical composition of the bars was determined by glow discharge surface analyser LECO GDS 750A.

Microstructure examination of the specimens before and after tensile testing was performed by optical microscopy (OM) using Olympus GX51 microscope and scanning electron microscopy (SEM) with JEOL JSM-6610 microscope. Samples for OM and SEM were prepared by grinding with water-resistant abrasive papers with grit size ranging from 320 to 2400 and polishing with diamond pastes with various grains size up to $1 \mu \mathrm{m}$. The polished samples were etched in a solution consisting of $90 \mathrm{ml}$ of $96 \%$ ethanol and $10 \mathrm{ml}$ $\mathrm{HNO}_{3}$. Quantitative metallography was performed on digital optical micrographs using computerised image analyser and software SigmaScanPro.

Vickers microhardness measurements were carried out on polished and slightly etched samples at an applied load of $0.05 \mathrm{~N}$ and hold time of $10 \mathrm{~s}$ using microhardness tester FM-ARS 9000. Vickers hardness measurements were performed at an applied load of $298 \mathrm{~N}$ and hold time of $10 \mathrm{~s}$ using HPO $250 \mathrm{KR} / \mathrm{AQ}$ machine.

Room temperature tensile tests were carried out on threaded-head cylindrical specimens with a gauge diameter of $5 \mathrm{~mm}$ and gauge length of $30 \mathrm{~mm}$ at an initial strain rate of $1 \times 10^{-3} \mathrm{~s}^{-1}$ using Zwick/Roell Z100 tensile machine. The tensile tests were run until achieving selected deformations of 3, 6 and $10 \%$ and until specimen fracture. Elongation of the specimens was measured by extensometer MAYTEC touching the surface of the gauge section of the specimen. The deformation was also measured by a $3 \mathrm{D}$ digital

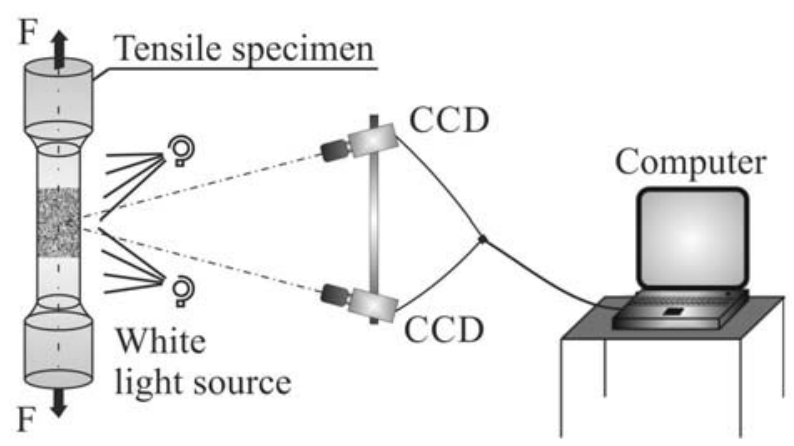

Fig. 1. Schema of 3D digital image correlation method for the cylindrical tensile specimen.

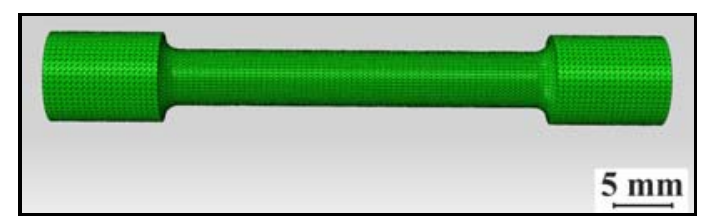

Fig. 2. Finite element meshes for the cylindrical type of tensile specimen.

image correlation (DIC) method using two high-speed CCD cameras MCR-2048-53 2/3", as seen in Fig. 1.

The DIC method is based on the application of a stochastic pattern on the surface of the test object [5-7]. The surface pattern on tensile specimens was achieved by spraying a white based colour on the gauge section and spattering random black colour speckles on this basic layer. The applied DIC software divides the observed surface area into smaller areas called facets. The speckles copy deformations of the surface and they move together with the surface during tensile deformation. Displacement of the speckles and corresponding strains were calculated through correlation of corresponding facets on digital frame at the position before and after the deformation [8-10]. The system realises calculations with the assistance of correlation algorithm based on the pseudo-affine coordinate transformation of object points from one picture to the second one. If displacement vectors of all points and reference outline are known, then it is possible to calculate the strain fields. The strain fields can be determined directly by the derivative of displacement of adjoining points or by analysis of local facets bending [2].

The strains fields were also determined by finite element analysis (FEA) with Abaqus software [11-14]. The input data required for the elastic-plastic model in FEA Abaqus such as the Young's modulus, true stress, true plastic strain, and strain hardening curve of the studied steel were determined experimentally by the tensile tests. The values of Poisson's ratio and true 
Ta ble 1. Chemical composition of the studied steel (wt.\%)

\begin{tabular}{ccccccccccc}
\hline $\mathrm{C}$ & $\mathrm{Mn}$ & $\mathrm{Si}$ & $\mathrm{P}$ & $\mathrm{S}$ & $\mathrm{Cr}$ & $\mathrm{Ni}$ & $\mathrm{Cu}$ & $\mathrm{Co}$ & $\mathrm{Zr}$ & $\mathrm{Al}$ \\
\hline 0.33 & 0.85 & 0.26 & 0.021 & 0.016 & 0.089 & 0.039 & 0.053 & 0.018 & 0.024 & 0.013 \\
\hline
\end{tabular}
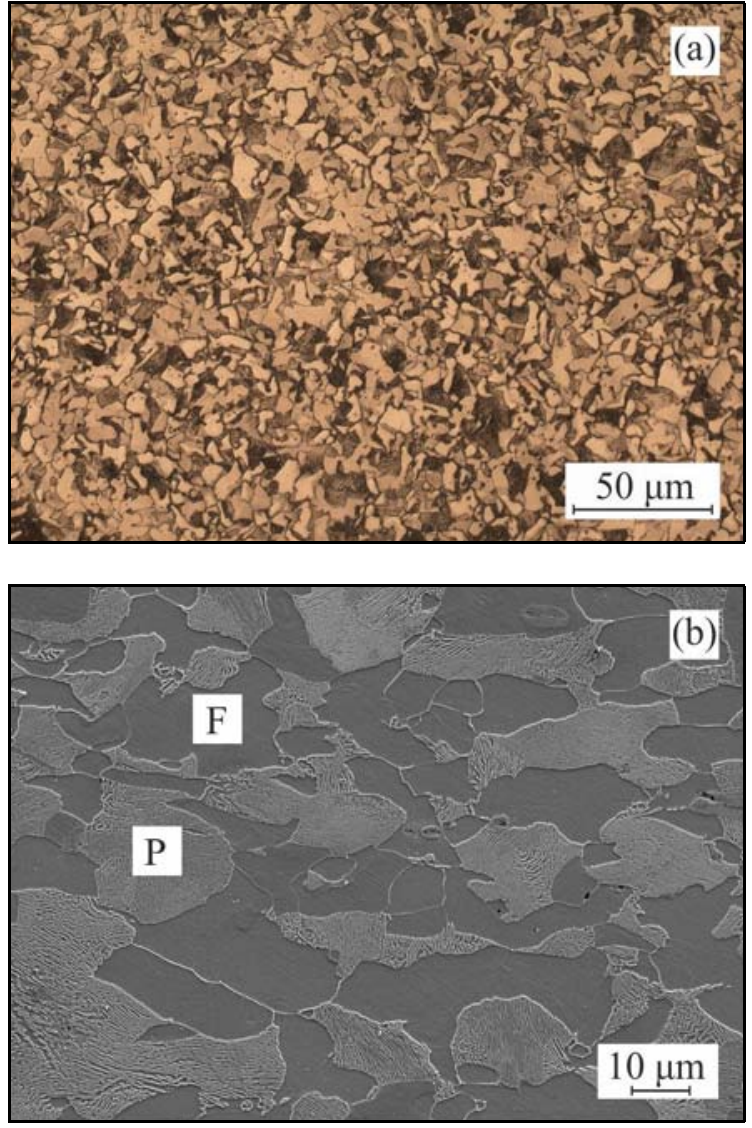

Fig. 3. The typical microstructure of the studied ferriticpearlitic steel before tensile testing: (a) equiaxed grain structure, OM; (b) ferritic grains (F) and pearlitic colonies (P), SEM.

plastic Poisson's ratio were taken from the literature [15]. Figure 2 shows the finite element meshes generated on the tensile specimens. For 3D analysis, the second-order tetrahedral elements (the element type in Abaqus: C3D10) with a size of $0.5 \mathrm{~mm}$ were used for the selected geometry of tensile specimens.

\section{Results and discussion}

\subsection{Microstructure before tensile testing}

Table 1 summarises the results of GDS measurements of the chemical composition of the as-received bars. The studied material fulfils the requirements for the medium carbon steel according to the Slovak technical standards STN 411550 (DIN 17100 St 55) and is frequently used for the production of seamless tubes. Figure 3a shows the typical grain structure on a longitudinal section of the specimen before tensile testing. Similar grain morphology was also observed on transversal sections of the specimens indicating negligible anisotropy of the tensile specimens. The structure of the specimens was characterised by a shape factor, size and volume fraction of coexisting ferritic grains $(F)$ and pearlitic colonies $(P)$, as shown in Fig. $3 \mathrm{~b}$. The shape factor $F$ indicating the circularity of the grains on $2 \mathrm{D}$ projection is defined in the form

$$
F=\frac{4 \pi A}{P^{2}}
$$

where $A$ and $P$ is the area and perimeter of the ferritic grain or pearlitic colony, respectively. The statistical analysis of experimental data leads to medium shape factor of $(0.515 \pm 0.007)$ and $(0.537 \pm 0.006)$ for the ferritic grains and pearlitic colonies, respectively. The shape factor about 0.5 indicates slightly elongated grains and colonies, which can be characterised by their major and minor axis. The measured length of the major and minor axis of the ferritic grains and pearlitic colonies (minimum 800 measurements of each type) can be well fitted by a log-normal distribution function, as seen in Fig. 4. The major mean length of the ferritic grains and pearlitic colonies is determined to be $(9.4 \pm 0.2) \mu \mathrm{m}$ and $(8.5 \pm 0.2) \mu \mathrm{m}$, respectively. The minor mean length of the ferritic grains and pearlitic colonies is measured to be $(5.0 \pm 0.2) \mu \mathrm{m}$ and $(4.6 \pm 0.2) \mu \mathrm{m}$, respectively. The correlation coefficients of these fits are better than $r^{2}=0.97$. The volume fraction of the ferritic grains and pearlitic colonies is determined to be $(51.1 \pm 0.8)$ vol. $\%$ and $(48.9 \pm 0.9)$ vol.\%, respectively, which corresponds very well to the volume fraction of coexisting phases according to the $\mathrm{Fe}-\mathrm{Fe}_{3} \mathrm{C}$ phase diagram [16].

\subsection{Vickers microhardness, hardness, and tensile properties}

Statistical evaluations of Vickers microhardness measurements (minimum 30 measurements for each region) leads to average values of $(2.37 \pm 0.02) \mathrm{GPa}$ and $(2.80 \pm 0.02) \mathrm{GPa}$ for the ferritic grains and pearlitic colonies, respectively. The average value of Vickers hardness is measured to be $(2.44 \pm 0.04) \mathrm{GPa}$.

Figure 5 shows the typical tensile engineering stress-strain curves of the specimens tested to strains of 3,6 and $10 \%$. The average values of offset $0.2 \%$ 
Table 2. Room-temperature tensile properties

\begin{tabular}{|c|c|c|c|c|c|c|c|}
\hline \multirow{2}{*}{$\begin{array}{c}\text { Deformation } \\
\quad(\%)\end{array}$} & \multicolumn{3}{|c|}{ Tensile curves } & \multicolumn{2}{|c|}{ Measurements on specimens } & \multicolumn{2}{|c|}{ DIC measurements } \\
\hline & $\begin{array}{c}\mathrm{YS} \\
(\mathrm{MPa})\end{array}$ & $\begin{array}{c}\text { UTS } \\
(\mathrm{MPa})\end{array}$ & $\begin{array}{c}A \\
(\%)\end{array}$ & $\begin{array}{c}Z \\
(\%)\end{array}$ & $\begin{array}{c}A \\
(\%)\end{array}$ & $\begin{array}{c}Z \\
(\%)\end{array}$ & $\begin{array}{c}A \\
(\%)\end{array}$ \\
\hline 3 & $639 \pm 4$ & - & $3.0 \pm 0.0$ & $1.9 \pm 0.1$ & $3.0 \pm 0.0$ & $1.8 \pm 0.1$ & $3.0 \pm 0.0$ \\
\hline 6 & $628 \pm 2$ & $819 \pm 1$ & $6.0 \pm 0.1$ & $9.7 \pm 0.8$ & $6.0 \pm 0.3$ & $10.3 \pm 0.7$ & $5.9 \pm 0.1$ \\
\hline 10 & $628 \pm 3$ & $810 \pm 6$ & $10.8 \pm 0.6$ & $36.9 \pm 0.8$ & $10.9 \pm 0.8$ & $36.6 \pm 0.8$ & $10.8 \pm 0.5$ \\
\hline Fracture & $632 \pm 5$ & $815 \pm 5$ & $11.1 \pm 0.5$ & $38.2 \pm 0.7$ & $11.0 \pm 0.6$ & $38.1 \pm 0.7$ & $11.0 \pm 0.6$ \\
\hline
\end{tabular}

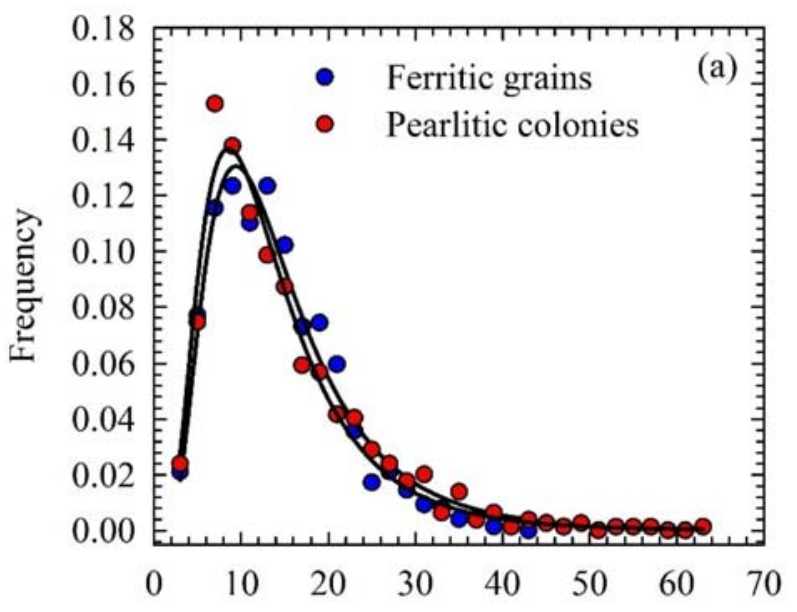

Length of major axis $(\mu \mathrm{m})$

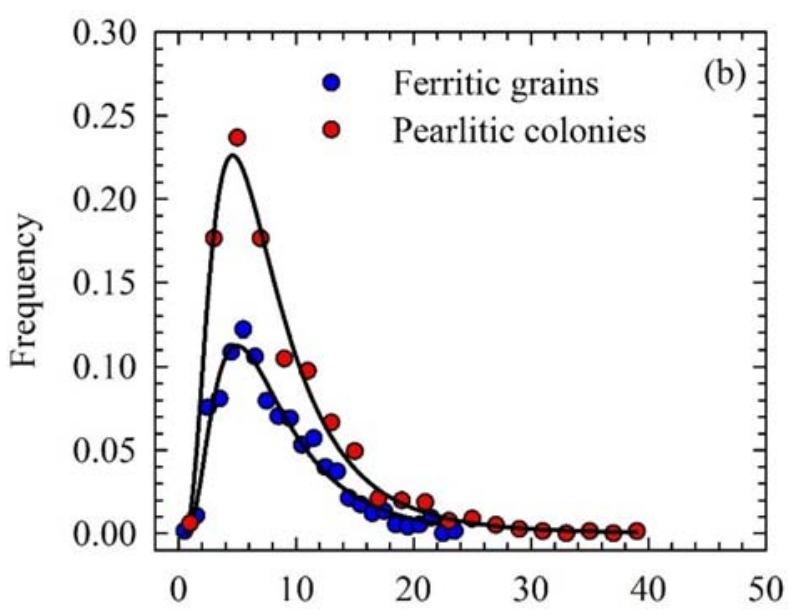

Length of minor axis $(\mu \mathrm{m})$

Fig. 4. Log-normal distribution curves of measured size of ferritic grains and pearlitic colonies: (a) major axis, (b) minor axis.

yield strength (YS), ultimate tensile strength (UTS), elongation $(A)$ and reduction of area $(Z)$ are summarised in Table 2. As can be seen in this table, plastic elongation to fracture measured by extensometer corresponds very well to the values measured manually

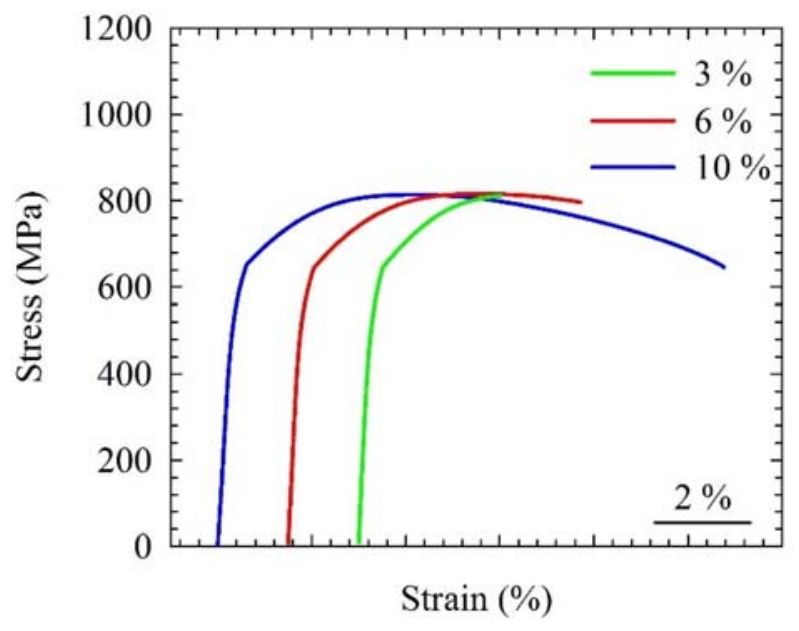

Fig. 5. Room temperature tensile stress-strain curves. The achieved plastic deformation is indicated in the figure.

on the specimens after tensile testing and by the DIC method using Mercury software. For the contraction, the values measured manually on the specimens correspond very well to those from the DIC measurements.

\subsection{Tensile deformation}

The direct manual measurements of elongation and contraction on the tensile specimens allow to achieve only discrete values after testing of the selected deformation or fracture. On the other hand, the DIC method equipped with video extensometer of Mercury RT software provides large elongation and contraction data set allowing mapping whole tensile deformation process. Also, the Mercury RT software enables to calculate strain fields from the experimentally measured displacement fields during the entire tensile tests.

Figure 6 shows the typical pictures of the tensile specimen taken by the CCD cameras. The random black colour speckles pattern is very well visible on the gauge surface before and after tensile testing. The green line indicated along the gauge section of the specimen is drawn by video extensometer of Mercury RT software before tensile testing (Fig. 6a) followed the 

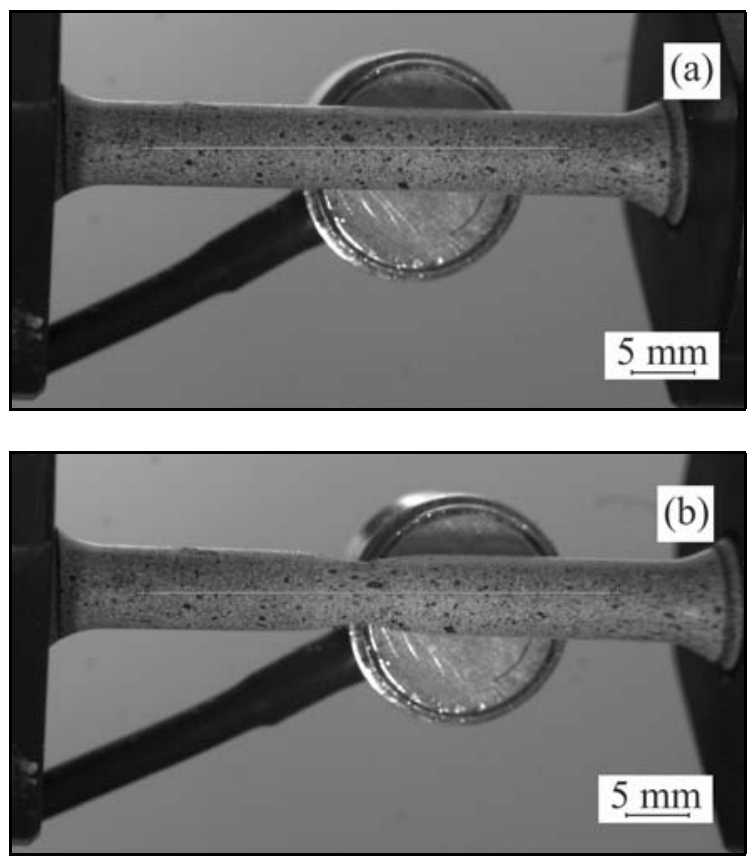

Fig. 6. Pictures of a tensile specimen taken by the CCD cameras: (a) before deformation, (b) after average tensile deformation of $10 \%$.

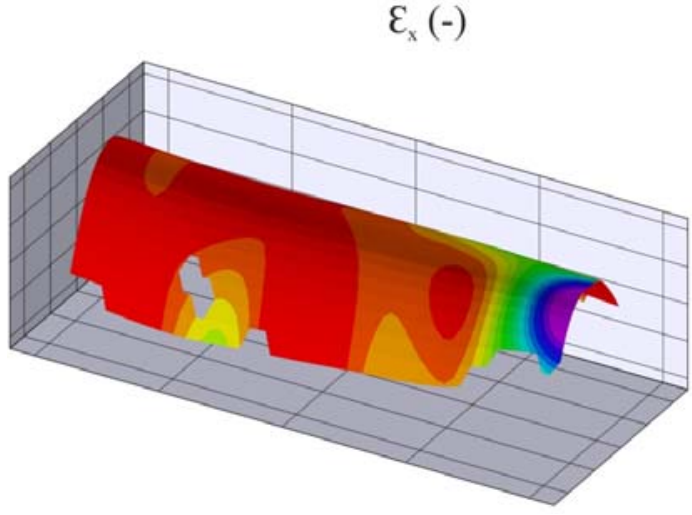

$\varepsilon_{\mathrm{x}}(-)$

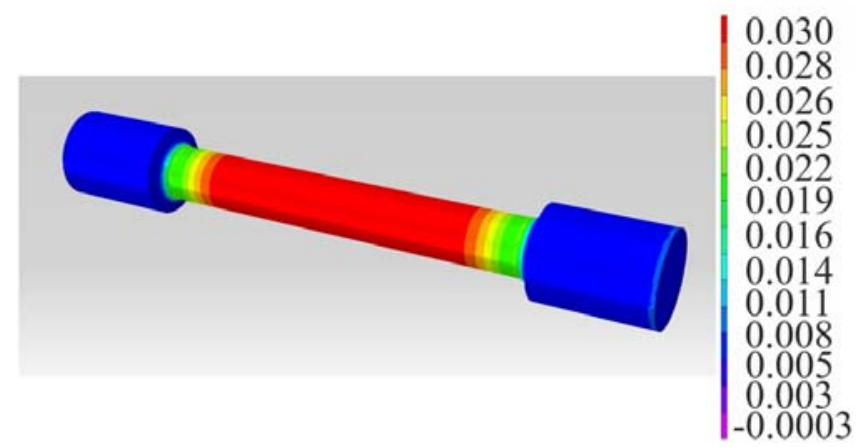

Fig. 7. Calculated $\varepsilon_{x}$ strain fields for the specimen tested to $3 \%$ average deformation: (a) DIC method; (b) FEA.

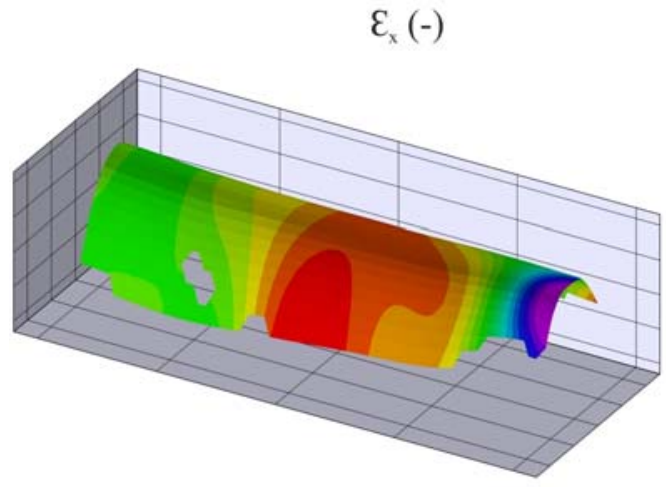

$\varepsilon_{\mathrm{x}}(-)$

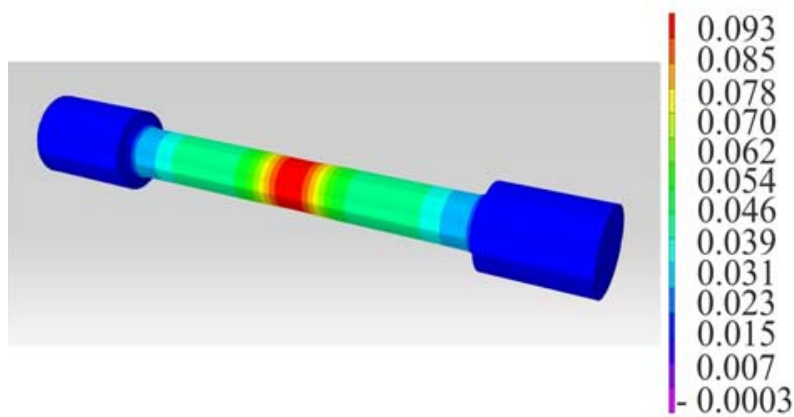

Fig. 8. Calculated $\varepsilon_{x}$ strain fields for the specimen tested to $6 \%$ average deformation: (a) DIC method; (b) FEA.

elongation of the specimen during whole deformation process (Fig. 6b). The specimen shows non-uniform deformation with the well developed necked region after an average plastic deformation of $10 \%$, as seen in Fig. 6b.

Figure $7 \mathrm{a}$ shows the $3 \mathrm{D}$ visualisation of $\varepsilon_{x}$ strain fields measured by the DIC method on the surface of the specimen tested to $3 \%$ average deformation. The surface deformation is nearly uniform along the entire gauge section because small variations in strain ranging from 0.023 to 0.030 are negligible. Figure $7 \mathrm{~b}$ shows a $3 \mathrm{D}$ visualisation of $\varepsilon_{x}$ strain fields calculated by FEA indicating the uniform deformation of the gauge section. Very good agreement between strain fields calculated by the experimental DIC method and those resulting from the FEA calculations indicates the appropriateness of the Abaqus input data experimentally determined for the studied steel in the frame of this work. It should be noted that so-called uniform deformation at the macro level along the entire gauge section is highly non-uniform in terms of the microstructure. As shown by Cuddy and Nabil Bassim [17], dislocation structure reflects the inhomogeneous distribution of strain with more advanced development within the ferrite grains.

Figure $8 \mathrm{a}$ shows the $3 \mathrm{D}$ visualisation of $\varepsilon_{x}$ strain 


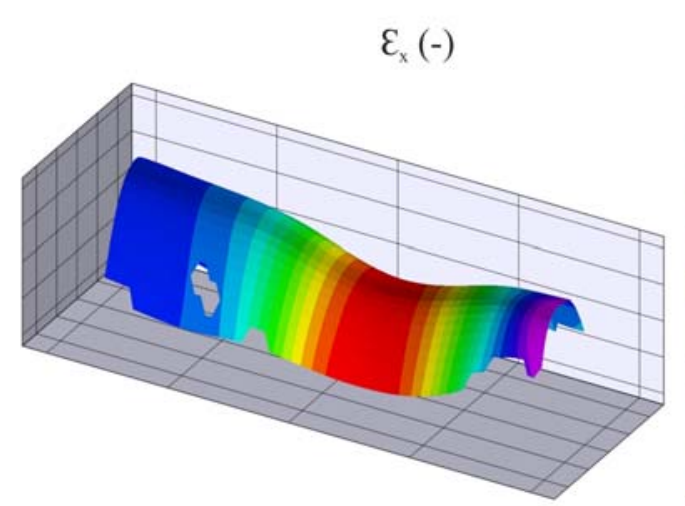

(a)
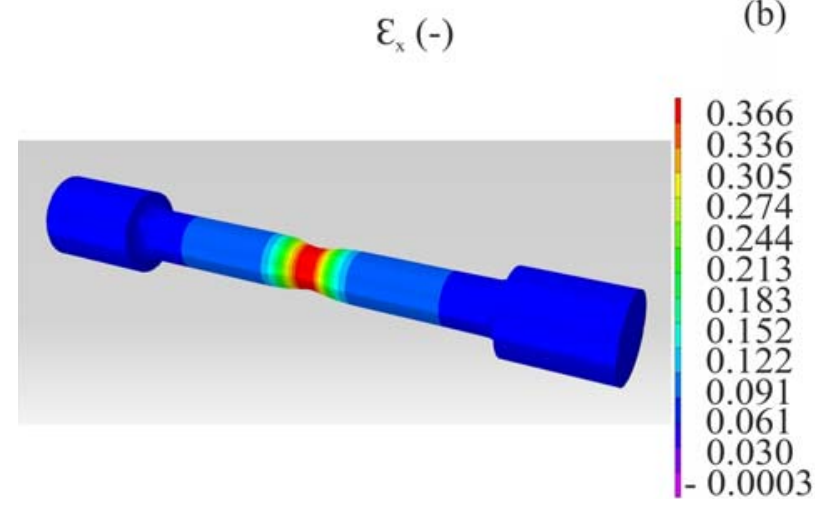

Fig. 9. Calculated $\varepsilon_{x}$ strain fields for the specimen tested to $10 \%$ average deformation: (a) DIC method; (b) FEA.

fields measured by the DIC method on the surface of the specimen tested to $6 \%$ average deformation. This figure clearly indicates localised non-uniform deformation with early stages of necking characterised by maximum local deformation of $9.3 \%$. Figure $8 \mathrm{~b}$ shows a $3 \mathrm{D}$ visualisation of $\varepsilon_{x}$ strain fields calculated by FEA. Intuitive prediction of failure of the specimen indicated by non-uniform strain distribution within the gauge section by FEA corresponds very well to the experimental DIC calculations.

Figure 9a shows the 3D visualisation of $\varepsilon_{x}$ strain fields measured by the DIC method on the surface of the specimen tested to $10 \%$ average deformation. Figure $9 \mathrm{~b}$ shows a $3 \mathrm{D}$ visualisation of $\varepsilon_{x}$ strain fields calculated by FEM. Both Figs. 9a and 9b clearly indicate highly non-uniform deformation with extensive necking and achieving maximum local strains up to $36.6 \%$ in the necked region. Much smaller deformation ranging from 3 to $6 \%$ is calculated outside the necked region.

\subsection{Work hardening behaviour}

Measurements of elongation and contraction by the DIC method during entire tensile deformation enable to calculate true stresses and true strains along the

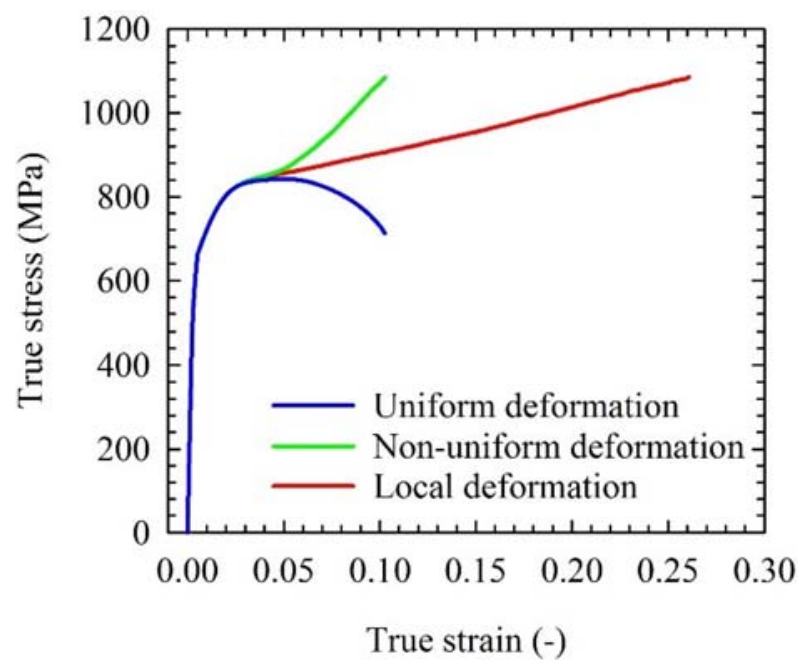

Fig. 10. An example of true stress-true strain curves calculated assuming a uniform, non-uniform and local deformation of the tensile specimen.

gauge section of the specimens. Figure 10 shows the true stress and true strain curves calculated assuming a uniform, non-uniform and local deformation of the gauge section. For the uniform deformation (blue curve in Figure 10), the true stresses $\sigma_{\mathrm{t}}$ are calculated assuming uniform contraction along the entire gauge section as a function of the elongation. The average true strains $\varepsilon_{\mathrm{t}}$ are calculated from the engineering strains $\varepsilon$ according to the relationship $\varepsilon_{\mathrm{t}}=\ln (1+\varepsilon)$. For the non-uniform deformation, the true stresses are calculated assuming the experimentally measured contractions (maximum values) by the DIC method and assuming the calculated average true strains $\varepsilon_{\mathrm{t}}$. For the local deformation, the true stresses are calculated assuming measured contractions (maximum values) by the DIC method and local true strains calculated from the average local engineering strains measured for the necked region.

The work hardening behaviour of the studied steel can be described by Hollomon's equation in the form [18]:

$$
\sigma_{\mathrm{t}}=K \varepsilon_{\mathrm{t}}^{n},
$$

where $n$ is the work hardening exponent and $K$ is the strength coefficient. The work hardening exponent indicates work hardening ability of the material and the higher $n$ indicates higher work hardening rate. Taking the true stresses between the yield strength and ultimate tensile strength and true strains, regression analysis yields $n=0.14 \pm 0.01$ and $K=(1395 \pm 85) \mathrm{MPa}$. The value of $n=0.14$ is comparable with the values ranging from 0.12 to 0.40 reported by Zuo et al. [19] for medium carbon low-alloy steels. For the strength coefficient, the values ranging from 1500 to $7000 \mathrm{MPa}$ were reported for Fe-0.37C-0.48Si-0.86Mn-1.67Cr-0.36Mo- 

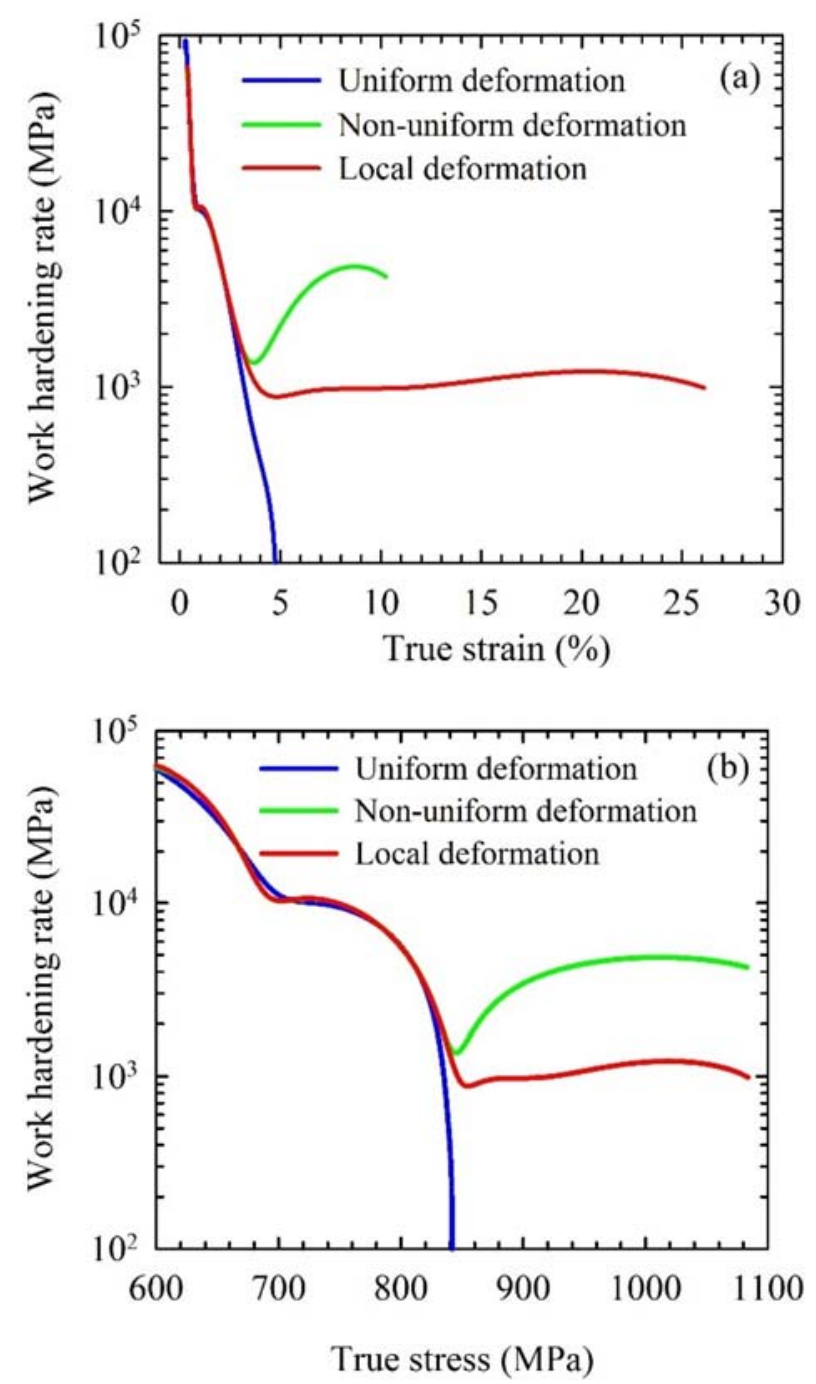

Fig. 11. Dependence of work hardening rate calculated assuming a uniform, non-uniform and local deformation of the tensile specimen on (a) true strain, (b) true stress.

$-0.015 \mathrm{P}-0.003 \mathrm{~S}$ (wt.\%) steel subjected to heat treatments [19].

Figure 11 shows evolution of work hardening rate $\Theta$ defined as

$$
\Theta=\frac{\mathrm{d} \sigma_{\mathrm{t}}}{\mathrm{d} \varepsilon_{\mathrm{t}}}
$$

Figure 11a shows the evolution of the work hardening rate with the true strain. It is clear from this figure that the evolution of the work hardening rate with the true strain is nearly the same for the uniform, nonuniform and local deformation up to true strains of about $3 \%$. At true strains higher than $3 \%$, the work hardening rate continuously decreases with increasing true strain up to the tensile fracture for the uniform deformation (blue curve). For the non-uniform deformation (green curve), the $\Theta$ increases with increasing $\varepsilon_{\mathrm{t}}$ after achieving true strain of about $5 \%$.
For the local deformation (red curve), the $\Theta$ decreases to a minimum value of about $870 \mathrm{MPa}$ at $\varepsilon_{\mathrm{t}}$ of about $5 \%$ and then varies in relatively narrow interval ranging from 850 to $1200 \mathrm{MPa}$ with increasing true strain up to tensile fracture. Figure 11b shows the dependence of the work hardening rate on the true stress. Four stages can be distinguished on the work hardening rate curves. During the stage I between 600 and $700 \mathrm{MPa}$, which corresponds to true strain ranging from 0.2 to $1.1 \%$, the strain hardening rate rapidly decreases from about $6 \times 10^{4}$ to $1 \times 10^{4} \mathrm{MPa}$ with increasing true strain. The stage II between 700 and $750 \mathrm{MPa}$, which corresponds to true strain ranging from 1.1 to $1.3 \%$, the strain hardening rate is nearly constant of about $1 \times 10^{4} \mathrm{MPa}$. The stage III between 750 and $850 \mathrm{MPa}$ corresponding to the true strain between 1 and $5 \%$ is characterised by a decrease of work hardening rate. The stage IV is observed only for the non-uniform (green curve) and local deformation (red curve) at true stresses higher than $850 \mathrm{MPa}$ and true strains higher than $5 \%$. The true strain of about $5 \%$ can be considered as the onset of plastic instability of the specimen. However, during the stage IV not only multiaxial stress conditions but also nucleation and growth defects such as cavities and cracks affect the work hardening behaviour in the necked region. It should be noted that three stages of the work hardening were reported by Zuo et al. [19] for medium carbon low-alloy steels analysed in the terms of uniform deformation. Each stage of the work hardening can be well related to the deformation of microstructure constituents (ferrite and pearlite) as shown for DP steels by N. Farabi et al. [20].

\subsection{Fractographic analysis}

The fracture surface shows mixed intergranular and transgranular ductile type of failure mode represented by microcavities, which are preferentially distributed within the ferritic grains. Typical SEM micrographs of the fracture surface are shown in Fig. 12a. The fracture surface contains basically dimples indicating typical ductile fracture caused by simple tensile loading and some cracks. Careful observations of longitudinal sections of tensile specimens indicate that the cavities are preferentially nucleated at ferritepearlite grain boundaries, as seen in Fig. 12b. The ferritic and pearlitic grains can be distinguished on the fracture surface. During tensile deformation, these cavities grow preferentially within the ferrite along the grain boundaries. The initiation and propagation of cracks within some pearlitic grains are also observed on longitudinal sections of tensile specimens in the vicinity of fracture surface. The size and shape of the dimples on fracture strongly depend on the coalescence of such cavities and transgranular propagation of cracks leading to the fracture of tensile specimens. 

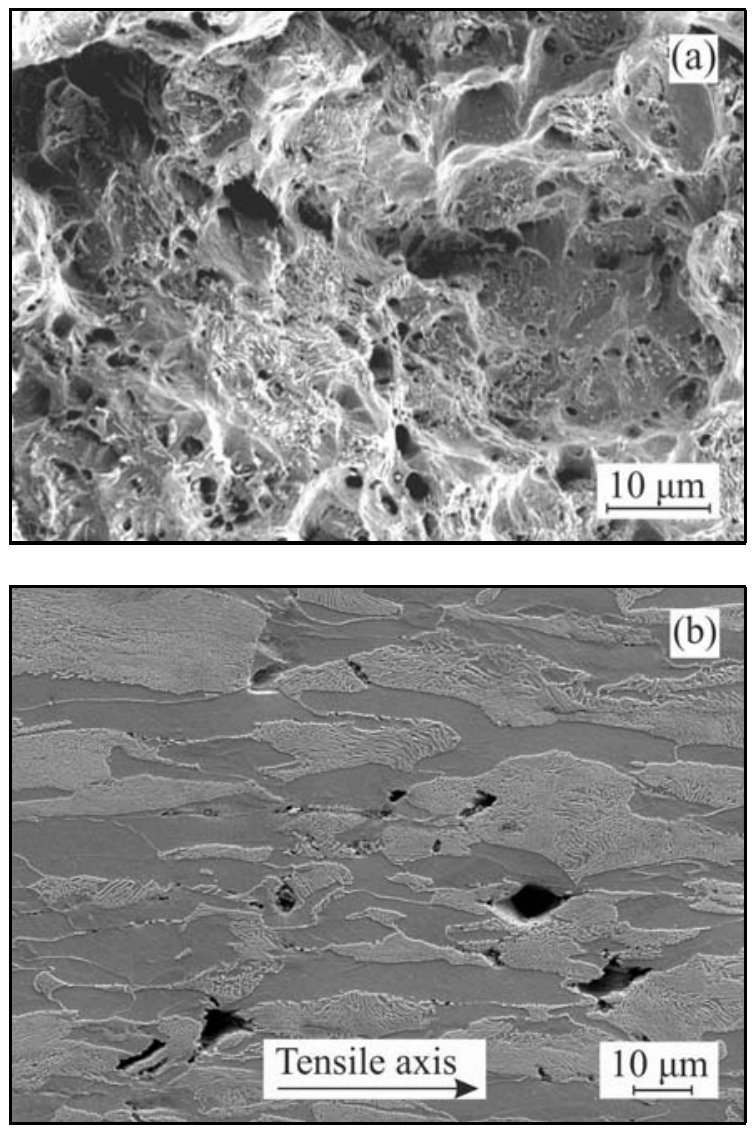

Fig. 12. Typical SEM micrographs of the tensile specimen after a fracture: (a) fracture surface, (b) longitudinal section in the vicinity of fracture surface.

\section{Conclusions}

The results of investigation of tensile deformation behaviour of ferritic-pearlitic steel using digital image correlation method can be summarised as follows:

1. The chemical composition, shape factor, mean values of the major and minor axis and volume fraction of ferritic grains and pearlitic colonies of tensile specimens are determined.

2. Room temperature tensile properties such as yield strength, ultimate tensile strength, elongation, and contraction are determined for selected batch of tensile specimens.

3. The strain fields determined by experimental 3D DIC method on the surface of cylindrical tensile specimens can be well compared with the $3 \mathrm{D}$ calculations of the strain fields performed by FEA using Abaqus input data for the studied steel measured in the frame of this work.

4. The work hardening exponent, strength coefficient, and work hardening rate are calculated for the studied steel. The strain hardening rate is evaluated assuming non-uniform and local deformation of tensile specimens using experimental data measured by the DIC method.
5. The fractographic analysis shows mixed intergranular and transgranular ductile types of fracture mode with cavities and secondary cracks. During tensile testing, the cavities are preferentially nucleated at ferrite-pearlite grain boundaries.

\section{Acknowledgements}

This work was financially supported by the Slovak Grant Agency for Science under the contract VEGA 2/0149/13, Slovak Research and Development Agency under the contract APVV-0434-10 and Competence Center for New Materials, Advanced Technologies and Energy ITMS 26240220073, supported by the Research and Development Operational Program funded by the European Regional Development Fund.

\section{References}

[1] Bhadeshia, H. K. D. H., Honeycombe, R.: Steels Microstructure and Properties. 3rd Edition. Oxford, Butterworth-Heinemann 2006.

[2] Huang, J., Shi, D. Q., Yang, X. G., Pan, B., Shi, H.: Science China Technological Sciences, 57, 2014, p. 1411. doi: $10.1007 / \mathrm{s} 11431-014-5555-8$

[3] Rossi, M., Pierron, F., Stamborská, M., Šimčák, F.: Experimental and Applied Mechanics, 4, 2013, p. 229. doi: 10.1007/978-1-4614-4226-4_27

[4] Hagara, M., Schrötter, M.: American Journal of Mechanical Engineering, 1, 2013, p. 185.

doi: 10.12691/ajme-1-7-6

[5] Stamborská, M., Mareš, V., Kvíčala, M., Horsák, L.: Kovove Mater., 52, 2014, p. 377.

doi: 10.4149/km_2014_6_377

[6] Schrötter, M., Hagara, M., Kalina, M.: Applied Mechanics and Materials, 611, 2014, p. 490. doi: 10.4028/www.scientific.net/AMM.611.490

[7] Simčák, F., Stamborská, M., Huňady, R.: CHEMICKÉ LISTY, 105, 2011, p. 564.

[8] Hua, T, Xie, H. M., Wang, S., Hu, Z., Chen, P., Zhang, Q.: Opt Laser Technol, 43, 2011, p. 9. doi:10.1016/j.optlastec.2010.04.010

[9] Gao, J., Shang, H.: Applied Optics, 48, 2009, p. 1371. doi: 10.1364/AO.48.001371

[10] Besnard, G., Hild, F., Roux, S.: Experimental Mechanics, 46, 2006, p. 789. doi: 10.1007/s11340-006-9824-8

[11] Garino, C. G., Gabaldón, F., Goicolea, J. M.: Finite Elements in Analysis and Design, 42, 2006, p. 1187. doi:10.1016/j.finel.2006.05.004

[12] Zeng, Z., Fatemi, A.: Journal of Strain Analysis, 36, 2001, p. 287. doi: 10.1243/0309324011514476

[13] Sethuraman, R., Viswanadha, G. S.: Int. J. Pres. Ves. Pip., 81, 2004, p. 313. doi:10.1016/i.ijpvp.2004.03.002

[14] Ince, A., Glinka, G.: J Strain Anal Eng, 48, 2013, p. 229. doi: $10.1177 / 0309324713477638$

[15] Cabezas, E. E., Celentano, D. J.: Mecánica Computacional, XXI, 2002, p. 854. doi:10.1016/S0168-874X(03)00096-9

[16] Shaposhnikov, N. G.: C-Fe Phase Diagram. ASM Alloy Phase Diagrams Database. Materials Park, ASM 
International 2008. http://www1.asminternational. org/asmenterprise/APD/ViewAPD.aspx?id=104069

[17] Cuddy, J., Bassim, M. N.: Mater. Sci. Eng. A, 113, 1989, p. 421. doi:10.1016/0921-5093(89)90329-8

[18] Hollomon, J. H.: Am. Inst. Min. Metall. Eng. Trans. Iron Steel Div., 162, 1945, p. 268.
[19] Zuoa, X., Chen, Y., Wang, M.: Materials Research, 15, 2012, p. 915. doi: 10.1590/S1516-14392012005000118

[20] Farabi, N., Chen, D. L., Zhou, Y.: JMEPEG, 21, 2012, p. 222. doi: $10.1007 / \mathrm{s} 11665-011-9865-8$ 